\title{
Maintenance monotherapy with Gemcitabine following cisplatin-based primary combination chemotherapy in surgically treated advanced urothelial carcinoma: A matched-pair single institution analysis
}

\author{
CHARIS KALOGIROU $^{1}$, ANDREY SVISTUNOV ${ }^{1}$, MARKUS KREBS ${ }^{1}$, EVA MARIA LAUSENMEYER ${ }^{2}$, \\ DANIEL VERGHO $^{1}$, HUBERTUS RIEDMILLER ${ }^{1}$ and ARKADIUS KOCOT ${ }^{1}$ \\ ${ }^{1}$ Department of Urology and Paediatric Urology, Julius Maximilians University Medical Center of Würzburg, \\ D-97080 Würzburg; ${ }^{2}$ Department of Urology, University of Regensburg, D-93053 Regensburg, Germany
}

Received October 23, 2015; Accepted January 13, 2016

DOI: $10.3892 / \mathrm{mco} .2016 .749$

\begin{abstract}
The role of maintenance therapy with Gemcitabine (GEM) following cisplatin-based combination chemotherapy $(\mathrm{CBCC})$ in patients with surgically treated advanced urothelial carcinoma (UC) remains to be fully elucidated. In the present case control study, a retrospective analysis was performed to evaluate the role of GEM monotherapy following surgical intervention for advanced UC. Between 1999 and 2013, 38 patients were identified with surgically treated advanced UC after having completed CBCC, who were additionally treated quarterly with two consecutive GEM $\left(1,250 \mathrm{mg} / \mathrm{m}^{2}\right)$ infusions as maintenance therapy. This collective was matched by propensity score matching to a control collective $(n=38)$ that received primary $\mathrm{CBCC}$ alone, and the overall survival (OS), cancer-specific survival (CSS) and progression-free survival (PFS) rates were determined for the two collectives using Kaplan-Meier estimates and the log-rank test. Regression analysis was performed using the Cox proportional hazards model. The median follow-up time
\end{abstract}

Correspondence to: Dr Charis Kalogirou, Department of Urology and Pediatric Urology, Julius Maximilians Medical Center of Würzburg, 6-8 Oberdürrbacher Street, D-97080 Würzburg, Germany

E-mail: kalogirou_c@ukw.de

Abbreviations: UC, urothelial carcinoma; LUT, lower urinary tract; UUT, upper urinary tract; OS, overall survival; CSS, cancer-specific survival; PFS, progression-free survival; CT, computed tomography; CBCC, cisplatin-based combination chemotherapy; GFR, glomerular filtration rate; GEM, Gemcitabine; GC, Gemcitabine/ cisplatin; MVAC, methotrexate (M), vinblastin (V), adriamycin (A) and cisplatin (C); MVEC, methotrexate (M), vinblastin (V), epirubicin (E) and cisplatin (C)

Key words: Gemcitabine, monotherapy, urothelial carcinoma, bladder cancer was 37 months (interquartile range: 9-148). Interestingly, patients treated with GEM following primary chemotherapy had a significantly improved outcome with respect to the 5-year OS (46.2 vs. $26.4 \%, \mathrm{P}=0.0314)$ and 5-year CSS (61.3 vs. $33.4 \%, \mathrm{P}=0.0386)$ rates. Notably, the 5 -year PFS rate did not differ between the two groups (10.3 vs. $16.1 \%$, $\mathrm{P}=0.134)$. It is proposed that additional GEM maintenance monotherapy is able to improve survival rates following primary CBCC in surgically treated patients with advanced $\mathrm{UC}$, suggesting a possible treatment option for patients with, e.g., unclear disease status, or those who would require an active maintenance therapy in the future. Prospective studies should further determine the impact of GEM monotherapy with respect to PFS rates in groups comprising larger numbers of patients.

\section{Introduction}

The efficient 'killer' in the form of advanced urothelial carcinoma (UC) is often underestimated at present: 5-year overall survival (OS) rates rarely exceed $40-50 \%$, even following surgical treatment and adjuvant chemotherapy, with disappointing progression-free survival (PFS) rates observed of 16 to 18 months $(1,2)$. Following the failure of primary cisplatin-based combination chemotherapy (CBCC), in general, there are limited choices for second- or third-line therapies (3). The aim of the present study was to offer a comparatively safe additional maintenance treatment for patients with stable disease following $\mathrm{CBCC}$, with the objective of improving survival rates and the time to first progression. Gemcitabine (GEM) was selected as the treatment for the present study. After having noted the promising response rates $(4,5)$ and encouraging case reports (6), together with low reported rates of adverse events (4-7), 38 patients, predominantly after having achieved stable disease post-chemotherapy, were treated quarterly with two consecutive injections of $1,250 \mathrm{mg} / \mathrm{m}^{2} \mathrm{GEM}$ as previously described (4). Subsequently, OS, cancer-specific survival (CSS) and PFS rates were determined, in comparison with 
a matched control group that received best supportive care alone.

\section{Patients and methods}

Patient characteristics and GEM monotherapy. The present study included 38 patients suffering from advanced UC of the lower or upper urinary tract, who were surgically treated by radical cystoprostatectomy with extended lymphadenectomy or nephroureterectomy between 1999 and 2013 at our institution (the 'maintenance collective'). Patients who did not receive surgical care prior to adjuvant chemotherapy were excluded from the study. Indications for adjuvant primary chemotherapy were an advanced pathological tumor stage of pT3 or above (8), positive nodal status $(\mathrm{N}+)$, lymphangiosis (L1) or haemangiosis (V1) carcinomatosa, or perineural invasion (Pn1). Following the completion of primary CBCC [a treatment of methotrexate, vinblastin, adriamycin and cisplatin, or methotrexate, vinblastin, epirubicin and cisplatin, or GEM and cisplatin (GC), administered in a standardized regimen (9)], patients were staged by performing computed tomography (CT; Siemens SOMATOM Force; Siemens Healthcare AG, Erlangen, Germany) of the thorax and the abdomen. Of the patients examined, 33 were staged stable disease $(n=30)$ or remission $(n=3)$, whereas five patients were staged as mixed response $(n=3)$ or progressive disease $(n=2)$. For one patient, no information concerning the disease status was available. Further follow-up was performed every four months by CT of the thorax and the abdomen, or by magnetic resonance imaging (MRI; Siemens MAGNETOM Prisma MRI Scanner, Siemens Healthcare AG) of the abdomen with simultaneous chest X-ray scanning (Philips Bucky DIAGNOST X-ray system; Philips Medical Systems, Hamburg, Germany). Progression of the disease was defined as the appearance of any new lesion and/or a $25 \%$ increase in the sum of the pre-existing lesions. The time to progression was defined as the time from the initial diagnosis to the first event of clinical progression.

After checking inclusion criteria [sufficient renal function, i.e., glomerular filtration rate $(\mathrm{GFR})>30 \mathrm{ml} / \mathrm{min}$, hepatic function and the haemogram], all the patients were offered GEM maintenance therapy, and written informed consent was obtained from all the patients. The patients were treated quarterly with two consecutive (on days 1 and 8 of the cycle) short, $30 \mathrm{~min}$ infusions of $1,250 \mathrm{mg} / \mathrm{m}^{2} \mathrm{GEM}$ on an outpatient basis. As supportive agents, the patients received $8 \mathrm{mg}$ orally administered ondansetron prior to the GEM infusion. Blood tests were performed by the general practitioner of the patients prior to and following the therapy to monitor adverse events. GEM monotherapy was discontinued in the event of a marked clinical progression with rapid decline of the general health status, and severe renal (GFR $<30 \mathrm{ml} / \mathrm{min}$ ) or hepatic ( $>2$-fold increase in the level of transaminases) dysfunction, in addition to severe anaemia, leukopenia or thrombopenia. Two patients discontinued GEM monotherapy for personal reasons. Following the discontinuation of GEM monotherapy due to progression of the disease, a further two patients received second-line treatment $\left[320 \mathrm{mg} / \mathrm{m}^{2}\right.$ vinflunine in one patient, and dose-reduced GC in the other (to $50 \%$ of the standard dose in the other patient]. There were no dose reductions of GEM when administered alone; in patients not qualifying for the full dose of the $\operatorname{drug}\left(1,250 \mathrm{mg} / \mathrm{m}^{2}\right)$, therapy was discontinued.

Data analysis, matching approach and the control collective. The present study made use of cases of patients with UC who had received surgical attention at the Departments of Urology and Paediatric Urology at the University Medical Center of Würzburg, and at the University of Marburg $(n=62)$ between 1993 and 2009. These patients receiving the best supportive care alone following first- or second-line chemotherapy were set as controls (the 'control collective'). To improve comparability, the GEM monotherapy patient collective $(n=38)$ was matched with 38 patients of the control collective by propensity score matching, using the bioconductor package 'MatchIt' for R, version 3.10 (the type of matching employed was the nearest neighbour method; http://www.r-project.org). The matching criteria were age at diagnosis, gender, location of the primary tumor (lower or upper urinary tract), the tumor/lymph node/metastasis (TNM) status [using the version of the TNM classification of the International Union Against Cancer revised in 2009 (8)] and the number of initial chemotherapy cycles. Kaplan-Meier estimates for the OS, CSS and PFS rates were realized with the open-source software "R", version 3.10, and the bioconductor package 'survival', as well as Cox proportional hazard models concerning CSS in the two collectives. The log-rank test was used to determine statistical differences between survival estimates, and Pearson's chi-squared test was used to realize intergroup comparisons. On occasions where two means of normally distributed data were to be compared, the two-sided unpaired student's t-test was used. More than two group means were differentiated by analysis of variance with post hoc testing (Tukey's test) where significant differences occurred. $\mathrm{P}<0.05$ was taken to indicate a statistically significant difference.

\section{Results}

The characteristics of the patient collectives are shown in Tables I and II. Following matching, no statistical differences were identified with respect to the age at diagnosis, gender, site of the primary tumor, the TNM status or the number of initial chemotherapy cycles. The follow-up was also comparable. Patients in the monotherapy collective received GC more often than patients in the control collective did $(\mathrm{P}<0.01)$. However, no significant statistical differences were identified regarding the efficacy of primary chemotherapy $(\mathrm{P}=0.06)$.

Regarding oncological outcomes, 22 patients died in the maintenance collective, 21 mortalities of which were cancer-specific. In the control collective, mortality was recorded in 29 patients and cancer-specific mortality in 23 patients, results which were statistically significant $(\mathrm{P}=0.0076$ for mortality from any cause, and $\mathrm{P}=0.046$ for cancer-specific mortality). Clinical failure was noted in 18 patients in the maintenance collective and in 21 patients in the control collective, results which were comparable $(\mathrm{P}=0.33)$.

The 5-year OS rate was significantly higher $(49.2 \%)$ in the maintenance collective compared with the control collective 
Table I. Patient characteristics showing unmatched collectives.

\begin{tabular}{|c|c|c|c|}
\hline Variable & 'Maintenance collective' & 'Control collective' & P-value \\
\hline Number of patients & 38 & 62 & \\
\hline Follow-up (months) & $9-148(37)$ & $1-99(35)$ & 0.062 \\
\hline Age & $33-80(66)$ & $26-84(67)$ & 0.201 \\
\hline Initial cycles & $1-6(3.1)$ & $1-6(2.8)$ & 0.25 \\
\hline Mono cycles & $1-48(5.1)$ & NA & \\
\hline \multicolumn{4}{|l|}{ Sex } \\
\hline Male & $30(78.9 \%)$ & $48(77.4 \%)$ & \\
\hline Female & $8(21.1 \%)$ & $14(22.5 \%)$ & 0.84 \\
\hline \multicolumn{4}{|l|}{ Localization } \\
\hline Lower urinary tract & $29(76.3 \%)$ & $42(67.8 \%)$ & \\
\hline Upper urinary tract & $9(23.7 \%)$ & $20(32.2 \%)$ & $<0.01$ \\
\hline \multicolumn{4}{|l|}{$\mathrm{pT}^{\mathrm{a}}$} \\
\hline Tis & $1(2.6 \%)$ & $0(0.0 \%$ & \\
\hline 1 & $5(13.1 \%)$ & $3(4.8 \%)$ & \\
\hline $2 \mathrm{a}$ & $4(10.5 \%)$ & $6(9.7 \%)$ & \\
\hline $2 b$ & $4(10.5 \%)$ & $7(11.3 \%)$ & \\
\hline $3 a$ & $9(23.7 \%)$ & $11(17.7 \%)$ & \\
\hline $3 b$ & $4(10.5 \%)$ & $20(32.3 \%)$ & \\
\hline $4 a$ & $8(21.0 \%)$ & $14(22.6 \%)$ & $p T 1+2$ vs.pT3+4 \\
\hline $4 b$ & $0(0.0 \%)$ & $1(1.6 \%)$ & 0.04 \\
\hline \multicolumn{4}{|l|}{$\mathrm{pN}^{\mathrm{a}}$} \\
\hline $0 / x$ & $11(28.9 \%)$ & $3(4.8 \%)$ & \\
\hline 1 & $11(28.9 \%)$ & $20(32.3 \%)$ & \\
\hline 2 & $13(34.2 \%)$ & $36(58.1 \%)$ & $p N O+1$ vs. $p N 2+3$ \\
\hline 3 & $3(7.8 \%)$ & $3(48.4 \%)$ & $<0.01$ \\
\hline \multicolumn{4}{|l|}{$\mathrm{pM}^{\mathrm{a}}$} \\
\hline 0 & $32(84.2 \%)$ & $55(88.7 \%)$ & \\
\hline 1 & $6(15.7 \%)$ & $7(11.3 \%)$ & $<0.01$ \\
\hline
\end{tabular}

${ }^{\mathrm{a}}$ Further details for the pathological (p) staging categories, and the pT, pN and pM subcategories, may be found in (8). NA, not applicable.

(26.5\%, log-rank $\mathrm{P}=0.0314)$, in addition to the 5-year CSS rate $(61.3 \%$ in the maintenance collective vs. $33.4 \%$ in the control collective; log-rank $\mathrm{P}=0.0386$ ). The 5-year PFS rate did not differ significantly between the control and the maintenance collectives $(10.3 \%$ in the maintenance collective vs. $16.1 \%$ in the control collective; log-rank $\mathrm{P}=0.134$ ). Fig. 1 summarizes these results. Prognostic factors that influence the CSS rate were also identified in all patients by compiling Cox proportional hazard models. In addition, the present study revealed that the efficacy of primary chemotherapy $(\mathrm{P}=0.0168)$ and GEM maintenance therapy $(\mathrm{P}=0.0396)$ were independent prognostic factors in uni- and multivariate analysis (Table III). All the other examined factors (age, gender, the location of primary tumor, the TNM status and the number of initial chemotherapy cycles) were not significantly predictive of the CSS rate.

\section{Discussion}

Uro-oncologists find that their available options soon become limited with respect to advancing UC following primary CBCC (3). Rapid rates of clinical failure (on average, 16-18 months) quickly exhaust secondary or third-line treatment options (2); what remains is supportive therapy (e.g. radiation therapy or polymodal pain management) and, ultimately, following palliative care, mortality.

To improve the survival rate and the time to first progression, maintenance therapy is required. GEM monotherapy has been assessed in specific solid and haematological cancers (10-12) and, although no established maintenance therapy for UC is available at present, predominantly Japanese researchers have gained experience in this cancer entity with GEM monotherapy, demonstrating comparably low, 
Table II. Patient characteristics showing matched collectives.

\begin{tabular}{|c|c|c|c|}
\hline Variable & 'Maintenance collective' & 'Control collective' & P-value \\
\hline Number of patients & 38 & 38 & \\
\hline Follow-up (months) & $9-148(37)$ & $1-97(34.5)$ & 0.562 \\
\hline Age (years) & $33-80(66)$ & $28-83(68)$ & 0.24 \\
\hline Initial cycles & $1-6(3.1)$ & $1-5(2.4)$ & 0.06 \\
\hline Mono cycles & $1-48(5.1)$ & NA & \\
\hline \multicolumn{4}{|l|}{ Gender } \\
\hline Male & $30(78.9 \%)$ & $29(76.3 \%)$ & \\
\hline Female & $8(21.1 \%)$ & $9(23.7 \%)$ & 0.7 \\
\hline \multicolumn{4}{|l|}{ Localization } \\
\hline Lower urinary tract & $29(76.3 \%)$ & $30(78.9 \%)$ & \\
\hline Upper urinary tract & $9(23.7 \%)$ & $8(21.1 \%)$ & 0.69 \\
\hline \multicolumn{4}{|l|}{$\mathrm{pT}^{\mathrm{a}}$} \\
\hline Tis & $1(2.6 \%)$ & $0(0.0 \%$ & \\
\hline 1 & $5(13.1 \%)$ & $0(0.0 \%)$ & \\
\hline $2 \mathrm{a}$ & $4(10.5 \%)$ & $6(15.8 \%)$ & \\
\hline $2 b$ & $4(10.5 \%)$ & $5(13.2 \%)$ & \\
\hline $3 a$ & $9(23.7 \%)$ & $9(23.7 \%)$ & \\
\hline $3 b$ & $4(10.5 \%)$ & $16(42.1 \%)$ & \\
\hline $4 a$ & $8(21.0 \%)$ & $0(0.0 \%)$ & $p T 1+2$ vs.pT $3+4$ \\
\hline $4 b$ & $0(0.0 \%)$ & $1(2.6 \%)$ & 0.18 \\
\hline \multicolumn{4}{|l|}{$\mathrm{pN}^{\mathrm{a}}$} \\
\hline $0 / \mathrm{X}$ & $11(28.9 \%)$ & $3(7.9 \%)$ & \\
\hline 1 & $11(28.9 \%)$ & $13(34.2 \%)$ & \\
\hline 2 & $13(34.2 \%)$ & $22(57.9 \%)$ & $p N 0 / X+1$ vs. pN $2+3$ \\
\hline 3 & $3(7.8 \%)$ & $0(0.0 \%)$ & 0.135 \\
\hline \multicolumn{4}{|l|}{$\mathrm{pM}^{\mathrm{a}}$} \\
\hline 0 & $32(84.2 \%)$ & $34(89.5 \%)$ & \\
\hline 1 & $6(15.7 \%)$ & $4(10.5 \%)$ & 0.29 \\
\hline \multicolumn{4}{|c|}{ Efficacy of primary chemotherapy } \\
\hline Regression & $3(7.9 \%)$ & $1(2.6 \%)$ & \\
\hline Stable & $30(78.9 \%)$ & $23(605 \%)$ & \\
\hline Progression & $2(5.2 \%)$ & $3(7.9 \%)$ & \\
\hline Mixed response & $3(7.9 \%)$ & $3(7.9 \%)$ & reg+stable vs.prog+mixed \\
\hline $\mathrm{N} / \mathrm{A}$ & $1(2.6 \%)$ & $8(21.1 \%)$ & 0.06 \\
\hline \multicolumn{4}{|c|}{ Type of primary chemotherapy } \\
\hline $\mathrm{GC}$ & $35(92.2 \%)$ & $20(52.6 \%)$ & \\
\hline MVAC/MVEC & $1(2.6 \%)$ & $18(47.4 \%)$ & \\
\hline Other & $2(5.2 \%)$ & $0(0.0 \%)$ & $<0.01$ \\
\hline \multicolumn{4}{|c|}{ Adverse events monotherapy } \\
\hline Yes & $3(7.8 \%)$ & NA & \\
\hline No & $35(92.2 \%)$ & NA & NA \\
\hline \multicolumn{4}{|c|}{ Discontinuation of monotherapy } \\
\hline Personal & $2(5.2 \%)$ & NA & \\
\hline Side effects & $3(7.8 \%)$ & NA & NA \\
\hline \multicolumn{4}{|l|}{ Second-line therapy } \\
\hline Yes & $2(5.2 \%)$ & $1(2.6 \%)$ & \\
\hline No & $36(94.8 \%)$ & $37(97.4 \%)$ & 0.243 \\
\hline
\end{tabular}

${ }^{a}$ Further details for the pathological (p) staging categories, and the pT, pN and pM subcategories, may be found in (8). GC, Gemcitabine and cisplatin; MVAC, methotrexate, vinblastin, adriamycin and cisplatin; MVEC, methotrexate, vinblastin, epirubicin and cisplatin; NA, not applicable. 
Table III. Regression analysis.

\begin{tabular}{|c|c|c|c|c|c|c|}
\hline \multirow[b]{2}{*}{ Variable } & \multicolumn{2}{|c|}{ Univariate } & \multicolumn{4}{|c|}{ Multivariate } \\
\hline & $\chi^{2}$ & P-value & Coefficient & HR & CI 95\% (low-high) & P-value \\
\hline Age & 2.6 & 0.1066 & & & & \\
\hline Sex & 0.78 & 0.377 & & & & \\
\hline Location (lut/uut) & 0.27 & 0.6043 & & & & \\
\hline Initial cycles (<3 vs. $>3$ ) & 1.31 & 0.2527 & & & & \\
\hline \multicolumn{7}{|l|}{ Efficacy of primary } \\
\hline chemotherapy & 5.72 & 0.01681 & 0.8865 & 2.4270 & $0.41-5.14$ & 0.0207 \\
\hline GEM maintenance & 4.24 & 0.03957 & 0.6756 & 1.9653 & $0.51-3.78$ & 0.0434 \\
\hline $\mathrm{pT}^{\mathrm{a}}(\mathrm{pT} 1+2$ vs. $\mathrm{pT} 3+4)$ & 3.4 & 0.06539 & & & & \\
\hline $\mathrm{pN}^{\mathrm{a}}(\mathrm{pN} 0+1$ vs. $\mathrm{pN} 2+3)$ & 0.47 & 0.4918 & & & & \\
\hline $\mathrm{pM}^{\mathrm{a}}$ & 0.18 & 0.6728 & & & & \\
\hline
\end{tabular}

${ }^{\mathrm{a}}$ Further details for the pathological (p) staging categories, and the pT, $\mathrm{pN}$ and $\mathrm{pM}$ subcategories, may be found in (8). CI, confidence interval; lut, lower urinary tract; uut, upper urinary tract; GEM, Gemcitabine.
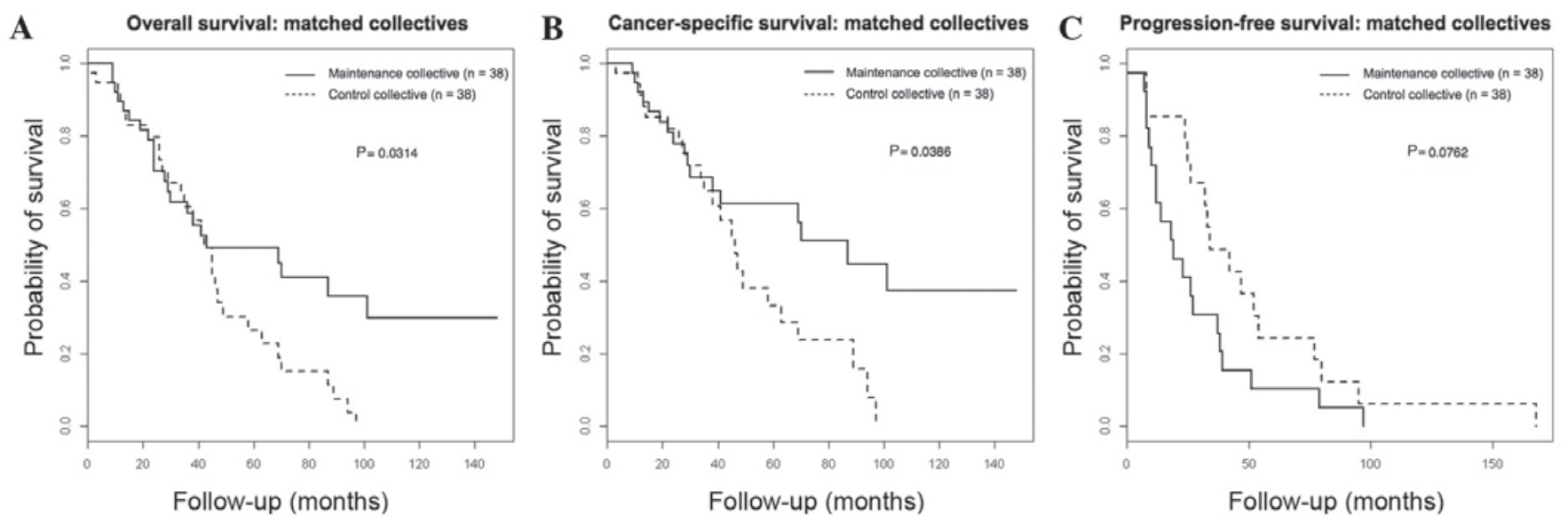

Figure 1. Kaplan-Meier estimates for (A) overall, (B) cancer-specific and (C) progression-free survival for the Gemcitabine maintenance collective (solid line, $n=38$ ) in comparison with the matched control collective (dashed line, $n=38$ ). The log-rank P-values are indicated on the right-hand side of the panels.

although potentially promising response rates when used as a monotherapy alone, Following primary $\mathrm{CBCC}$, a novel study published by Muto et al (7) demonstrated that GEM appeared to have a prognostic benefit in an UC patient collective $(n=33)$ of palliative, non-surgically treated patients. The authors showed marked advantages in the maintenance group with respect to CSS and PFS rates compared with a control group receiving best supportive care alone, accompanied by a low rate of adverse events.

This feature provided the foundational basis for the present study. Knowing that, after disease stabilization following primary $\mathrm{CBCC}$, the time to first progression rarely exceeds 24 months even in surgically treated patients with advanced UC (2), it became desirable to study the oncological effects of a GEM monotherapy in this particular patient group. In agreement with the study by Muto et al (7), a marked improvement in CSS and OS rates for the GEM maintenance patients was observed in the present study. It was also demonstrated that the efficacy of primary CBCC and GEM maintenance therapy were independent prognostic factors for the CSS rate in all patients. However, in contrast to Muto et al (7), presence of visceral metastasis was not identified as a prognosticator of note for the patients in the present study, predominantly due to the limited number of cases. Additionally, and again in contrast to Muto et al (7), the PFS rate was not affected by GEM monotherapy in the patient collective in the present study. Since GEM is a drug which the majority of the patients in the maintenance collective will have received prior to the study (GC is considered standard care at our institution), it may be hypothesized that the survival benefits observed in the present study are not the result of the prolonged exposure to the drug itself, but are due to a selection bias resulting from the retrospective nature of the study. 
However, it is reasonable to surmise that more than merely a selection bias accounted for the particular observations in the present study. Adjuvant therapies prolonging the OS and CSS rates, while having no effect upon the PFS rate, are well known in the treatment of other cancer entities, predominantly vaccination studies in prostate cancer, but also including studies in haematological malignancies $(13,14)$. Explanations for these effects may include delayed drug response, as well as changes in immunological and/or tumor-biological features. As GEM is known for changing humoral as well as tumor immunology in solid cancers (15-17), a combination of immunological and tumor-biological modifications in UC leading to the observed survival benefits in the maintenance group in the present study would be imaginable. However, the underlying molecular mechanisms leading to a positive response towards GEM monotherapy in UC have yet to be fully elucidated, and should therefore be explored in future studies for alleviated and personalized patient selection regarding this type of maintenance therapy (e.g., establishment of predictive biomarkers for the GEM treatment response). This consideration is highly supported by one particular patient of the present maintenance collective: After having received an unpleasant diagnosis in the year 2002 at the age of 49 with a pT3b pN3 M0 UC following anterior exenteration and creation of an ileocoecal pouch, the patient enjoys good health with no detectable sign of the disease in May 2015 in the 48th cycle of GEM monotherapy. Prior to monotherapy, the patient had received three cycles of GC, and was staged stable disease thereafter. At present, neither primary CBCC nor monotherapy have elicited any adverse effects with respect to either haematological effects or a decline of renal function in the patient.

Another aspect of GEM monotherapy as a maintenance treatment concept is the incorporation of this additive therapy regime with reference to vinflunine being approved for second-line chemotherapy following CBCC (18). We consider that GEM monotherapy (with its low demands on renal function and favourable adverse event rates) could be scheduled either prior to or following Vinflunine therapy, although this question will need to be definitively addressed through comprehensive and prospective clinical studies.

There were limitations to the present study. Being a retrospective analysis, sampling bias must be considered. Additionally, it has to be acknowledged that information concerning the efficacy of primary CBCC was not available in $\sim 20 \%$ of the control collective. Furthermore, in view of the comparatively small number of patients, changes in the time to progression, as previously mentioned, will need to be examined in prospective studies to come, including larger patient groups.

Despite these limitations, the present study indicates that GEM monotherapy is a good choice for young, surgically treated UC patients with stable disease, in the search for an active maintenance therapy following primary $\mathrm{CBCC}$; in our experience, such a therapy often encourages patients mentally, thereby improving the quality of life (and ultimately, as demonstrated in the present study, the survival rate).

In conclusion, additional GEM maintenance therapy may improve the survival rate in stable-disease, surgically treated patients with UC following primary platinum-based chemo- therapy, and should therefore be validated in prospective studies.

\section{Acknowledgements}

The present study was supported by the Open Access Publication Fund of the University of Wuerzburg.

\section{References}

1. Advanced Bladder Cancer (ABC) Meta-analysis Collaboration: Neoadjuvant chemotherapy in invasive bladder cancer: Update of a systematic review and meta-analysis of individual patient data advanced bladder cancer (ABC) meta-analysis collaboration. Eur Urol 48: 202-205; discussion 205-206, 2005.

2. Freiha F, Reese J and Torti FM: A randomized trial of radical cystectomy versus radical cystectomy plus cisplatin, vinblastine and methotrexate chemotherapy for muscle invasive bladder cancer. J Urol 155: 495-499; discussion 499-500, 1996.

3. Sonpavde G, Sternberg CN, Rosenberg JE, Hahn NM, Galsky MD and Vogelzang NJ: Second-line systemic therapy and emerging drugs for metastatic transitional-cell carcinoma of the urothelium. Lancet Oncol 11: 861-870, 2010.

4. Albers P, Siener R, Härtlein M, Fallahi M, Haeutle D, Perabo FG, Steiner G, Blatter J and Müller SC; German TCC Study Group of the German Association of Urologic Oncology: Gemcitabine monotherapy as second-line treatment in cisplatin-refractory transitional cell carcinoma-prognostic factors for response and improvement of quality of life. Onkologie 25: 47-52, 2002.

5. Akaza H, Naito S, Usami M, Miki T, Miyanaga $N$ and Taniai $H$; Japanese Gemcitabine Study Group: Efficacy and safety of gemcitabine monotherapy in patients with transitional cell carcinoma after Cisplatin-containing therapy: A Japanese experience. Jpn J Clin Oncol 37: 201-206, 2007.

6. Matsushima M, Kikuchi E, Masugi Y, Akita H, Miyajima A, Jinzaki $M$ and Oya M: Efficacy and safety of gemcitabine monotherapy in an elderly patient with penile metastasis from bladder carcinoma: A case report. Int Canc Conf J 2, 4, 255-260, 2013.

7. Muto S, Abe H, Noguchi T, Sugiura SI, Kitamura K, Isotani S, Ide H, Yamaguchi R, Kamai T and Horie S: Maintenance monotherapy with gemcitabine after standard platinum-based chemotherapy in patients with advanced urothelial cancer. Int J Urol 22: 490-494, 2015.

8. Cheng L, Montironi R, Davidson DD and Lopez-Beltran A: Staging and reporting of urothelial carcinoma of the urinary bladder. Mod Pathol 22: S70-S95, 2009.

9. von der Maase H, Hansen SW, Roberts JT, Dogliotti L, Oliver T, Moore MJ and Kerbrat P: Gemcitabine and cisplatin versus methotrexate, vinblastine, doxorubicin, and cisplatin in advanced or metastatic bladder cancer: Results of a large, randomized, multinational, multicenter, phase III study. J Clin Oncol 18: 3068-3077, 2000.

10. Karasek P, Skacel T, Kocakova I, Bednarik O, Petruzelka L, Melichar B, Bustova I, Spurny V and Trason T: Gemcitabine monotherapy in patients with locally advanced or metastatic pancreatic cancer: A prospective observational study. Expert Opin Pharmacother 4: 581-586, 2003.

11. Ricci S, Antonuzzo A, Galli L, Tibaldi C, Bertuccelli M, Lopes Pegna A, Petruzzelli S, Algeri R, Bonifazi V, Fioretto ML, et al: Gemcitabine monotherapy in elderly patients with advanced non-small cell lung cancer: A multicenter phase II study. Lung Cancer 27: 75-80, 2000.

12. Duvic M, Talpur R, Wen S, Kurzrock R, David CL and Apisarnthanarax N: Phase II evaluation of gemcitabine monotherapy for cutaneous T-cell lymphoma. Clin Lymphoma Myeloma 7: 51-58, 2006.

13. Madan RA, Gulley JL, Fojo T and Dahut WL: Therapeutic cancer vaccines in prostate cancer: The paradox of improved survival without changes in time to progression. Oncologist 15: 969-975, 2010.

14. Hernandez-Ilizaliturri FJ and Czuczman MS: Therapeutic options in relapsed or refractory diffuse large B-cell lymphoma. Part 1. Current treatment approaches. Oncology (Williston Park) 23: 546-553, 2009. 
15. Soeda A, Morita-Hoshi Y, Makiyama H, Morizane C, Ueno H, Ikeda M, Okusaka T, Yamagata S, Takahashi N, Hyodo I, et al: Regular dose of gemcitabine induces an increase in CD14+ monocytes and CD11c+ dendritic cells in patients with advanced pancreatic cancer. Jpn J Clin Oncol 39: 797-806, 2009.

16. Suzuki E, Kapoor V, Jassar AS, Kaiser LR and Albelda SM: Gemcitabine selectively eliminates splenic Gr-1+/CD11b+ myeloid suppressor cells in tumor-bearing animals and enhances antitumor immune activity. Clin Cancer Res 11: 6713-6721, 2005.

17. Nowak AK, Robinson BW and Lake RA: Gemcitabine exerts a selective effect on the humoral immune response implications for combination chemo-immunotherapy. Cancer Res 62: 2353-2358, 2002 .
18. Bellmunt J, Théodore C, Demkov T, Komyakov B, Sengelov L, Daugaard G and Delgado FM: Phase III trial of vinflunine plus best supportive care compared with best supportive care alone after a platinum-containing regimen in patients with advanced transitional cell carcinoma of the urothelial tract. J Clin Oncol: 27, 4454-4461, 2009. 